\section{CONSTRUCTION AND EVALUATION OF INTERLEUKIN 3 (IL3)-ZETAKINE REDIRECTED CYTOLYTIC T CELLS FOR THE TREATMENT OF CD123 EXPRESSING ACUTE MYELOID LEUKEMIA}

Rebecca Moeller*, Julian Scherer, Sadik Kassim. Vor Biopharma, Cambridge, MA, USA

Background Acute Myeloid Leukemia (AML) is an aggressive bone marrow malignancy, characterized by the presence of leukemic blasts in the peripheral blood of patients. Poor AML prognoses $^{1}$ are largely attributable to high rates of disease relapse, of which CD123 + leukemic stem cells (LSCs) are the primary cause. ${ }^{2}{ }^{3} \mathrm{CD} 123$, the alpha-chain of the IL3 cytokine receptor, ${ }^{6}$ has been identified as a favorable therapeutic AML target, overexpressed in both LSCs and blasts. ${ }^{4} 5$ We sought to direct $\mathrm{T}$ cells to CD123 + AML cells via cell surface tethered IL3 (termed "IL3-zetakine"). ${ }^{7}$ The use of a zetakine instead of a chimeric antigen receptor (CAR) construct enables structure-guided site-directed mutagenesis to increase binding affinity and alter target cell signaling without detrimental $\mathrm{T}$ cell hyperactivation.

Methods Zetakine constructs were designed using IL3 sequences bound to a transmembrane domain and intracellular costimulatory and $\mathrm{CD} 3 \mathrm{z}$ signaling domains. The constructs were transduced into Jurkat cells with lentiviral vectors (LVV). T cell activation via CD69 expression was assessed via flow cytometry of sorted IL3 zetakine-positive Jurkat cells after coculture with MOLM13 AML cells. Lead constructs were selected based on initial transduction percentage and activation response. In vitro functionality of each IL3 zetakine was tested with LVV transduced primary $\mathrm{T}$ cells by flow cytometry.

Results Zetakine constructs yielded a wide range of transduction percentages in Jurkat cells $(0-98 \%)$ prior to sorting. In co-cultures with CD123 + MOLM13 AML cells, Jurkat cells expressing wildtype IL3 constructs lacking a costimulatory domain induced the highest level of CD69 expression $(18.7 \%$ $\mathrm{CD} 69+\mathrm{T}$ cells) in an antigen-specific manner (5.3-fold increase of CD69+ $\mathrm{T}$ cells over those cultured with MOLM13 CD123KO cells). The K110E mutant IL3 was reported to exhibit a 40 -fold increased affinity over wildtype, ${ }^{8}$ but it showed no detectable zetakine function. However, additional mutant IL3 zetakines increased Jurkat cell activation up to 5.8-fold. Antigen-specific increases in CD69, as well as CD25, surface expression were also observed with zetakinetransduced primary $\mathrm{T}$ cells co-cultured with MOLM13 cells, in addition to target cell killing comparable to antibody-based CD123CAR T-cells.

Conclusions This work establishes IL3 zetakines as a viable alternative to traditional CD123-targeted CAR constructs. Structure-guided IL3 zetakine mutants with altered affinity and activation profiles will further our understanding of CD123specific cytotoxicity modulation without inducing acute $\mathrm{T}$ cell hyperactivation and exhaustion. These results indicate the ability of IL3 zetakine-expressing T cells to kill CD123-expressing AML cells and illustrate the potential of this novel class of therapeutics.

\section{REFERENCES}

1. Ganzel C, et al. Very poor long-term survival in past and more recent studies for relapsed AML patients: the ECOG-ACRIN experience. American journal of hematology 2018:10.1002/ajh.25162.

2. Shlush $\mathrm{LI}$, et al. Tracing the origins of relapse in acute myeloid leukaemia to stem cells. Nature 2017;547(7661):104-108.

3. Hanekamp D, Cloos J, Schuurhuis GJ. Leukemic stem cells: identification and clinical application. International Journal of Hematology 2017;105(5):549-557.
4. Bras $A E$, et al. CD123 expression levels in 846 acute leukemia patients based on standardized immunophenotyping. Cytometry part B: Clinical Cytometry 2019;96 (2):134-142.

5. Sugita M, Guzman ML. CD123 as a therapeutic target against malignant stem cells. Hematology/Oncology clinics of North America 2020;34(3):553-564.

6. Mingyue $S$, et al. CD123: a novel biomarker for diagnosis and treatment of leukemia. Cardiovascular \& Hematological Disorders-Drug Targets 2019;19(3):195204.

7. Kahlon $\mathrm{KS}$, et al. Specific recognition and killing of glioblastoma multiforme by interleukin 13-zetakine redirected cytolytic T cells. Cancer Res 2004;64(24):91606

8. Bagley $\mathrm{CJ}$, et al. A discontinuous eight-amino acid epitope in human interleukin-3 binds the alpha-chain of its receptor. J Biol Chem 1996;271(50):31922-8.

http://dx.doi.org/10.1136/jitc-2021-SITC2021.871 